\title{
AGB Mass-Loss History \& Haloes Around Planetary Nebulae
}

\author{
Romano L.M. Corradi \\ Isaac Newton Group of Telescopes, La Palma, Canary Islands, Spain
}

Matthias Steffen \& Detlef Schönberner

Astrophysikalisches Institut Potsdam, Potsdam, Germany

Mario Perinotto

Dipartimento di Astronomia e Scienza dello Spazio, Firenze, Italy

\section{Introduction}

Ionized haloes around planetary nebulae (PNe) are a quite common phenomenon, being found in about $70 \%$ of the elliptical $\mathrm{PNe}$ for which adequately deep images exist (cf. Corradi, these proceedings). Physically, one has to distinguish between two different kind of haloes. $A G B$ haloes consist of photo-ionized matter still containing information about the mass loss history during the last thermal pulse cycle on the Asymptotic $G$ iant $B$ ranch. A second kind of halo may develop when recombination sets in as a consequence of the fast luminosity drop during the advanced post-AGB evolution. These recombination haloes, which can be confused with real AGB haloes on a pure morphological basis, are not a signature of an AGB mass-loss episode (Corradi et al. 2000).

As part of an effort to extract information about the final mass-loss episode on the AGB from modern deep imaging of PN haloes, we compare the properties of the rather big AGB halo around the double-shell PN NGC 6826 with results of hydrodynamical simulations (cf. Steffen \& Schönberner, these proceedings).

\section{Halo observations \& hydrodynamical modeling}

A very deep $\mathrm{H} \alpha+[\mathrm{NII}]$ image of NGC 6826 was obtained at the $2.5 \mathrm{~m}$ Isaac Newton Telescope of La Palma, from which the surface brightness profiles for the inner nebula and halo were extracted. These were compared with different sets of models covering the evolution of the stellar wind over several 100000 years of AGB and post-AGB evolution. A notable result of these simulations is that the structure of the resulting AGB halo depends not only on the mass-loss history but also on the outflow velocity on the AGB, which in turn depends on the chemical composition of the dust grains. For a given evolution of the stellar parameters and mass loss rate, oxygen-rich and carbon-rich wind envelopes develop in fact AGB haloes with significantly different surface brightness profiles.

A surprisingly good fit of the observed halo surface brightness profiles of NGC 6826 was achieved with a particular set of hydrodynamical models (Fig. 1), 

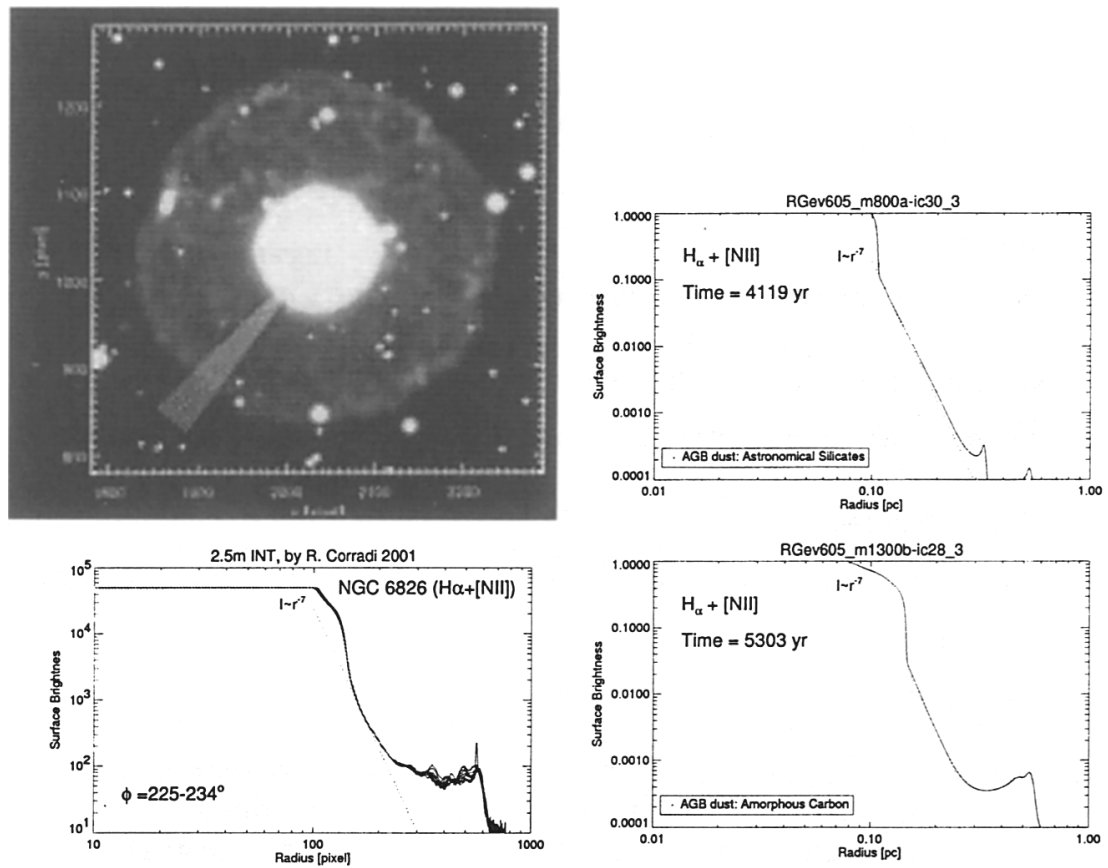

Figure 1. Left panels: Image of NGC 6826 (top), taken with the wide field camera at the prime focus of the $2.5 \mathrm{~m}$ Isaac Newton Telescope at La Palma, using a $100 \AA$ wide filter centered on $\mathrm{H}_{\alpha}$, together with the surface brightness profiles (bottom) measured along the radii indicated above. Right panels: Surface-brightness profiles $\left(\mathrm{H}_{\alpha}+\right.$ NII) of two hydrodynamical models, assuming an oxygen-rich (top) and a carbon-rich (bottom) AGB history, respectively.

indicating that (i) mass loss must increase substantially towards the end of the AGB phase $\left(I \propto r^{-7} \rightarrow \rho \propto r^{-4}\right.$, cf. Plait \& Soker 1990); (ii) the outer edge of the halo of NGC 6826 is defined by the luminosity minimum associated with the final helium-shell flash on the AGB, i.e. the kinematical age of the halo $\left(\sim 33000\right.$ yrs for $\left.d=1.1 \mathrm{kpc}, v_{\text {Halo }}=10 \mathrm{~km} / \mathrm{s}\right)$ measures the time elapsed since the last helium-shell flash, suggesting that the central star of NGC 6826 is most likely still powered by hydrogen burning; (iii) the surface brightness profile of the halo of NGC 6826 is better matched by the carbon-rich AGB wind model; indeed, the central star of NGC 6826 is known to be a carbon star $(C / O=4.5$ by number; Perinotto, 1991); (iv) it is not necessary to invoke wind interaction with the ISM to explain the typical limb-brightening of AGB the haloes.

\section{References}

Corradi, R., Schönberner, D., Steffen, M., Perinotto, M., 2000, A\&A 354, 1071

Perinotto, M., 1991, ApJS 76, 687

Plait, P., Soker, N., 1990, AJ 99, 1883 